Xavier Monnet

Jean-Louis Teboul

\section{End-tidal carbon dioxide and arterial pressure for predicting volume responsiveness by the passive leg raising test: reply to Piagnerelli and Biston}

Accepted: 28 March 2013

Published online: 25 April 2013

(C) Springer-Verlag Berlin Heidelberg and ESICM 2013

Monnet and Teboul are members of the Medical Advisory Board of Pulsion Medical Systems.

This reply refers to the comment available at: doi:10.1007/s00134-013-2913-0.

Dear Editor,

We thank Drs. Piagnerelli and Biston for their interest in our study "Endtidal carbon dioxide is better than arterial pressure for predicting volume responsiveness by the passive leg raising test" that was recently published in Intensive Care Medicine [1]. We also thank them for their valuable comments [2]. They point out that our inclusion criteria could represent limitations of the study that were not stated in the manuscript.

First, concerning the proportion of patients with cardiac arrhythmias and as recognized by Drs. Piagnerelli and Biston themselves, one advantage of the passive leg raising (PLR) test is that it remains valid in such a situation. As suggested by our colleagues in their letter, we compared the diagnostic ability of the changes in end-tidal carbon dioxide induced by the PLR test to predict fluid responsiveness in patients with and without arrhythmias. In the 11 patients (17\% of the population) with arrhythmias, the area under the receiver operating characteristics curve was $0.92 \pm 0.18$. In the 54 patients without arrhythmias, it was $0.97 \pm 0.02$. Thus, this study corroborates previous works, including a meta-analysis, in showing that the PLR test is valid in patients with an irregular cardiac rhythm.

Second, concerning norepinephrine administration, we agree that changing its dose might change cardiac preload and preload dependence, as recently demonstrated. This is the reason why the dose of norepinephrine was kept unchanged during the study time. Since norepinephrine was administered in almost all our patients (97\%), we cannot compare the PLR test validity in patients with and without vasoconstrictor administration.

Finally, we disagree with Drs. Piagnerelli and Biston when they suppose that our results might have been different in patients with higher tidal volumes. Indeed, though it is true that a low tidal volume invalidates indices of preload responsiveness based on the respiratory variations of hemodynamic signals, there is absolutely no reason why it should change the reliability of the PLR test. Obviously, the hemodynamic effects of PLR, which depend upon the transfer of blood from the leg splanchnic compartment, cannot be influenced by the tidal volume.

To conclude, we strongly believe that the results of our study may also apply in patients with cardiac arrhythmias, norepinephrine administration and non-low-tidal volume. As we did in the article [1], we emphasize that it might apply only to patients under mechanical ventilation without spontaneous breathing activity. Also, these results are limited to patients without any right ventricular failure and intra-abdominal hypertension since these two conditions were not investigated in the study.

\section{References}

1. Monnet X, Bataille A, Magalhaes E, Barrois J, Le Corre M, Gosset C, Guerin L, Richard C, Teboul JL (2013) End-tidal carbon dioxide is better than arterial pressure for predicting volume responsiveness by the passive leg raising test. Intensive Care Med 39:93-100

2. Piagnerelli M, Biston P (2013) End-tidal carbon dioxide and arterial pressure for predicting volume responsiveness by the passive leg raising test: a commentary. Intensive Care Med. doi:

10.1007/s00134-013-2913-0

X. Monnet ( $\bullet$ ) J.-L. Teboul AP-HP, Hôpitaux Universitaires Paris-Sud, Hôpital de Bicêtre, Service de réanimation médicale, Univ Paris-Sud, EA4533,

Le Kremlin-Bicêtre 94270, France e-mail: xavier.monnet@bct.aphp.fr 